\title{
Feasibility of 12 weeks of combined aerobic and resistance training in Indigenous Australian adults: A Phase I, single- group, pre-post intervention study
}

\author{
Tuguy Esgin*
}

Discipline of Exercise, Health and Performance, University of Sydney, Lidcombe, NSW, Australia School of Medical and Health Sciences, Edith Cowan University, Joondalup, WA, Australia

\begin{abstract}
Objectives: To investigate the feasibility and potential of an exercise program based on current recommendations for health to decrease cardiometabolic risk factors in Indigenous Australian adults in a metropolitan setting.

Design: Phase I, single-group, pre-post intervention study.

Methods: Sixteen previously inactive adults (55\% female; mean age 32 y) undertook supervised exercise involving aerobic and progressive resistance training for 3 days per week for 12 weeks.

Results: Eleven participants (69\%) completed the exercise intervention with high exercise adherence rates (92\% of sessions completed). Compared with baseline, there was a significant improvement with training in sub-maximal aerobic capacity (MD $5 \mathrm{ml} / \mathrm{kg} / \mathrm{min}, 95 \% \mathrm{CI} 3$ to 7 ), pulse wave velocity (MD -2 $\mathrm{m} / \mathrm{s}$; $95 \% \mathrm{CI}$ : -2.3 to -0.7 ), percentage body fat (MD $-2 \%$; CI: -3.4 to 0.2 ), bench press (MD $17 \mathrm{~kg}, 95 \% \mathrm{CI}: 10$ to 25 ), leg press (MD $67 \mathrm{~kg}$, CI: 36 to 110 ), waist circumference (MD $-3 \mathrm{~cm}, 95 \% \mathrm{CI}:-4.0$ to -1.0 ) and hip circumference (MD $-2 \mathrm{~cm}, 95 \% \mathrm{CI}:-4.4$ to -1.1$)$.
\end{abstract}

Conclusions: A supervised fitness centre based aerobic and resistance training program has the potential to reduce cardiovascular disease risk factors in Indigenous adults and can be delivered in a metropolitan gym environment.

\section{Introduction}

Avoidable, non-communicable chronic disease is the major cause of morbidity and mortality in Australian adults and is associated with a significant financial burden. In 2014 cardiovascular disease had the highest expenditure of any disease and was associated with an average hospitalisation cost of $\$ 9308$ per person [1]. In Australian and Torres Strait Islander communities; from here referred to as 'Indigenous communities', morbidity and mortality from non-communicable disease is significantly pronounced when compared with the nonIndigenous population [2] and heart disease and diabetes are the leading causes of death [3]. Costs associated with chronic disease may be increased for Indigenous versus non-Indigenous patients [4]. This may because social determinants of health, access to transport, lack of cultural competence are all barriers to the Indigenous communities accessing health services in a timely matter or complying with medical or health advice [5].

Lifestyle modification to lower cardiometabolic risk factors can significantly reduce morbidity and mortality and is a focus of chronic disease prevention strategies [6]. Estimates from non-Indigenous populations suggest that almost one third $(31 \%)$ of the burden of disease could be prevented by lifestyle modification [7]. For instance, interventions involving diet change, alcohol and smoking reduction have been shown to reduce a range of diseases from breast cancer [8] to diabetes [9], and to improve mental health outcomes [10].
For Indigenous Australians, the biggest health improvements can be achieved by reducing those risk factors which mediate the bulk of preventable chronic diseases including cardiovascular disease, diabetes and some cancers [6]. These well-established risk factors include dyslipidaemia, blood vessel dysfunction and hypertension, raised blood glucose/insulin and central obesity [11]. Simple lifestyle changes (dietary and physical activity) have been shown to improve plasma glucose, triglycerides and insulin in a Victorian Indigenous community [12].

Available evidence suggests that a short-term supervised exercise training intervention may improve fitness, waist circumference and systolic blood pressure in Indigenous adult woman [13] and that an intervention involving combined resistance training and sports participation can improve insulin sensitivity, body weight and waist circumference [14]. It is important to examine the efficacy of exercise for managing disease risk in Indigenous communities because physical activity was traditionally an important and essential part of Indigenous

${ }^{*}$ Correspondence to: Tuguy Esgin, Discipline of Exercise, Health and Performance, University of Sydney, Lidcombe, NSW, Australia School of Medical and Health Sciences, Edith Cowan University, Joondalup, WA, Australia, E-mail: tuguy1@gmail.com

Key words: aboriginal and torres strait islander, physical activity, muscle strength, central obesity, sedentary lifestyle

Received: February 12, 2019; Accepted: February 19, 2019; Published: February 22, 2019 
culture yet, when compared with non-Indigenous communities, sedentary behaviour may be higher and participation in sport and recreation lower in contemporary Indigenous society [15]. Physical activity/exercise is also a potentially low-cost and readily accessible intervention.

The aim of study was to investigate the feasibility of delivering an exercise program based on current recommendations for health [16] and its potential to decrease cardiometabolic risk factors in Indigenous Australian adults in a metropolitan setting.

\section{Method}

A Phase I, single-group, pre-post intervention study was carried out. This study examined the effect of 12 weeks of supervised aerobic and resistance exercise training in Indigenous Australian adults from the Perth Noongar community. Participants were measured preintervention (baseline) and after 12 weeks of the intervention. Postintervention measurements occurred at least three days but not more than seven days after the last exercise training session. All measurements were undertaken at the same site by the chief investigator.

Participants were recruited by meeting with key community members and organisation's and seeking their assistance in recruitment. One researcher (TE) was a member of the Perth Noongar community who facilitated links with the community and recruitment. To be eligible to participate individuals needed to: identify as being Australian Aboriginal or Torres Strait Islander; aged between 18-50 years; and provide clearance for participation from a medical practitioner. Pregnant women were not eligible to participate in the study.

Recruitment targeted Indigenous people from the Northern Perth region, either employed or studying at Edith Cowan University or living in the local area. However, the intervention was also delivered at a second site in Southern Perth after a Noongar Elder approached the researcher and requested that the intervention be conducted in the Southlake area.

The intervention involved accruing a minimum of 150 minutes of moderate intensity aerobic exercise each week and undertaking two or more sessions of group-based progressive resistance training (PRT), as per current American College of Sports Medicine (ACSM) guidelines [17]. All PRT exercise was supervised in a fitness centre environment, while aerobic exercise involved supervised and unsupervised activities. All participants were required to undertake at least two days of supervised group exercise, which included PRT. The fitness centre in the northern Perth suburbs had a female key advocate and the group was comprised exclusively of females, and the southern fitness centre had a male Elder as a key advocate and recruited mostly males. The researcher (TE) attended all fitness centre sessions with the participants at the Southlakes complex and another Exercise Physiologist attended the training sessions at the Wanneroo exercise complex and supervised the activity that took place as part of the session.

Aerobic exercise sessions involved walking, jogging, swimming or cycling (by personal preference) to accumulate the weekly target. All aerobic exercise was undertaken at a moderate intensity, equivalent to a perceived effort of $\sim 12-14$ on a scale of 20 (Borg), and was implemented by heart rate measurement or the "talk test". Participants used the built-in heart rate sensor that was associated with the machine they were using. The PRT intervention involved leg press, bench press, lat pull-down and shoulder press. Participants performed the exercises at an intensity of 10-12 repetition maximum (RM) (60\%) (the maximal weight that could be lifted for the specified number of repetitions) for the first 2 weeks, then 6-10RM (75\%) for weeks 3-12. Three sets of each exercise were completed with 60 - 90 s rest between sets, and 120-180 $\mathrm{s}$ interval between each exercise. Once participants could successfully perform a set of the target repetitions the training load was increased by approximately $5 \%$ to keep them within the target repetition range. 10-15 mins of warm-up preceded all exercise sessions via treadmill walking, cycle ergometer or cross-trainer.

Participants received a pass for entry into the fitness centre and were provided with an individualised program. Participants were also supplied with a diary and asked to record exercise training session information. Compliance was measured using the data compiled at the entry of the fitness centre.

Sub-maximal aerobic capacity was predicted using an AstrandRhyming test on a cycle ergometer (Repco, Exertech Ex-10, Huntingdale). A workload was selected that elicited a heart rate of between 130-150 bpm via telemetry (Polar, F1, Australia). Before the test commenced resting heart rate was recorded then at each minute for six minutes. After the participant had completed six minutes of cycling at the fixed workload, the difference in the heart rate at the end of the 5th and 6th minutes was compared. If the difference was greater than $5 \mathrm{bpm}$, the participant continued to pedal until steady state heart rate was achieved. This final average heart rate was recorded and $\mathrm{VO}_{2}$ maximum estimated (Astrand \& Rodahl, 1986).

Strength was measured by 1RM (Kraemer and Fry, 1995) bench and leg press. Before testing, the participants were familiarised with the procedures and warm-up, aerobic activity consisting of walking or jogging on a treadmill or stationary cycling for 10 minutes was completed followed by dynamic stretching.

Arterial occlusion was measured by placing a probe on the radial artery and reported as pulse wave velocity in $\mathrm{m} / \mathrm{s}$. Radial artery pressure waveforms of the same arm were sampled over 10 seconds with a Millar tonometer (SPC-301, Millar Instruments, Houston, United States of America). A generalised transfer function was applied to the radial artery waveform to obtain the pressure waveform at the ascending aorta to access arterial stiffness. Waveforms were then processed with dedicated software (SphygmoCor version 7, AtCor, Sydney, Australia). The software was used to calculate an averaged radial artery waveform and to calculate a corresponding central aortic pressure waveform employing previously validated functions. Aortic pressure waveforms were further analysed using the SphygmoCor software to determine the time to the peak/shoulder of the first and second pressure wave components (T1, T2) during systole. The pressure at the peak/ shoulder of the first component was identified as P1 height (outgoing pressure wave), and the pressure difference between this point and the maximal pressure during systole ( $\mathrm{P}$ or augmentation) was identified as the reflected pressure wave occurring during systole. Augmentation index (AIx), was defined as the ratio of augmentation to central pulse pressure, expressed as a percentage: Aix (P/ PP) 100, where $\mathrm{P}$ is pressure and $\mathrm{PP}$ is pulse pressure. This method has been validated against invasive techniques for determination of central BP and the augmentation index (AIx) is a marker of systemic arterial stiffness [18].

Anthropometry (waist circumference, hip circumference, weight) were measured. Height was measured to the nearest $0.1 \mathrm{~cm}$ using a stadiometer (SECA 700, Brooklyn, NY, USA). Circumferences at the waist and hip were measured to the nearest $0.1 \mathrm{~cm}$ at standard ISAK anatomical points using an anthropometric tape measure. Waist circumference was measured in a horizontal plane one $\mathrm{cm}$ above the iliac crest and hip circumference was assessed as the greatest 
circumference of the buttocks. Weight was measured to the nearest $0.1 \mathrm{~kg}$ using an electronic scale (AND TB: 200, Tokyo, Japan) in light clothing with shoes removed.

Whole body lean mass was measured using dual-energy X-ray absorptiometry (DEXA, Hologic Discovery A, Waltham, MA, USA).

Brachial blood pressure was measured according to the ASCOT protocol using a validated, semi-automated oscillometric device (Omron 705 CP, Omron, Japan). Before measurement, all participants rested for 5 minutes in a quiet room, after which time blood pressure was measured over the brachial artery 3 times at 5-minute intervals. The mean of the measurements was recorded as representative of brachial blood pressure.

Feasibility and participant characteristics are presented as mean (SD) and number (\%). For clinical outcomes, paired t-tests were conducted and the between-time differences presented as mean difference (95\% confidence interval), between Week 0 and Week 12 .

\section{Results}

Eleven previously inactive adults aged 32 (SD 7) years, of which 6 (55\%) were female; undertook supervised exercise involving 3 days per week of aerobic and progressive resistance training for 12 weeks.

Of the 37 participants that completed baseline testing, only 16 commenced the exercise intervention. There were numerous reasons why 21 participants never began the intervention. Unable to be contacted once the intervention started, moving to a new house and away from the intervention sites, work commitments, or no longer interested. 16 participants expressed an interest in participating in the fitness centre intervention, but only 11 commence the intervention with all 11 completing the study. There were several reasons for the initial 16 participants that were ready to start the gym intervention to be reduced to 11 . The researcher was unable to contact one participant using contact details provided after baseline assessments. A participant obtained a Fly in Fly Out mining job and had to withdraw from the study on the eve of its commencement. The $3^{\text {rd }}$ and $4^{\text {th }}$ persons were a mother and daughter who had shifted their place of residence. Due to the distance of travel involved, it would not have been practical for them to attend the gym. The $5^{\text {th }}$ participant was unable to meet the time commitments due to changing requirements of her academic studies (Figure 1).

Overall, the exercise intervention had high exercise adherence rates for the 11 participants who completed the intervention of over $94 \%$, with 33 out of a possible 36 sessions completed. This is based on participant attendance from start to the end of the intervention. Regarding adverse events, there were expected episodes of delayed onset of muscle soreness reported early in the intervention.

The mean time taken to collect measures was 70 min per participant and clinical measures were obtained for 11 participants at baseline and post exercise intervention.

The clinical outcomes for the 11 participants who completed the intervention are presented in (Table 1). Between pre-and post the training, $\mathrm{VO}_{2}$ maximum increased by $5 \mathrm{ml} / \mathrm{kg} / \mathrm{min}$ (95\% CI 3 to 7 ), $1 \mathrm{RM}$ bench press increased by $17 \mathrm{~kg}$ (95\% CI 10 to 25), 1RM leg press increased by $67 \mathrm{~kg}$ (CI 36 to 110), pulse wave velocity decreased by 1.5 $\mathrm{m} / \mathrm{s}$ (95\% CI 0.7 to 2.3 ). There was no statistically significant change in blood pressure.

There was a significant increase in aerobic fitness (mean difference $5 \mathrm{ml} / \mathrm{kg} / \mathrm{min}$; $95 \%$ CI 3 to 7 ). There was a significant increase in upper (mean difference $17.4 \mathrm{~kg} ; 95 \%$ CI 10.0 to 25.1 ) and lower (mean difference $73.4 \mathrm{~kg}$; 95\% CI 36.7 to 110.1 ) body strength with training. The exercise program resulted in a decrease in per cent body fat (mean difference $5.6 \%$; $95 \%$ CI -0.2 to -3.4 ) and a reduction in average waist (mean difference $3.7 \mathrm{~cm}$ : 95\% CI -1.1 to -6.3 ), hip circumference decrease (mean difference $2.4 \mathrm{~cm}$; $95 \%$ CI -1.1 to 4.4 ) and Waist to Hip ratio (WHR) (mean difference $0.1 ; 95 \%$ CI 0.0 to 0.1 ). Fat mass in the trunk region was not significantly changed (mean difference $1.1 \mathrm{~kg}$; $95 \%$ CI -0.5 to 2.8 ). Pulse wave velocity was significantly reduced with exercise training (mean difference $1.5 \mathrm{~m} / \mathrm{s} ; 95 \%$ CI 0.7 to -2.3 ) but there were no significant changes in any of the vascular outcomes (Table 2).

37 participants initially tested

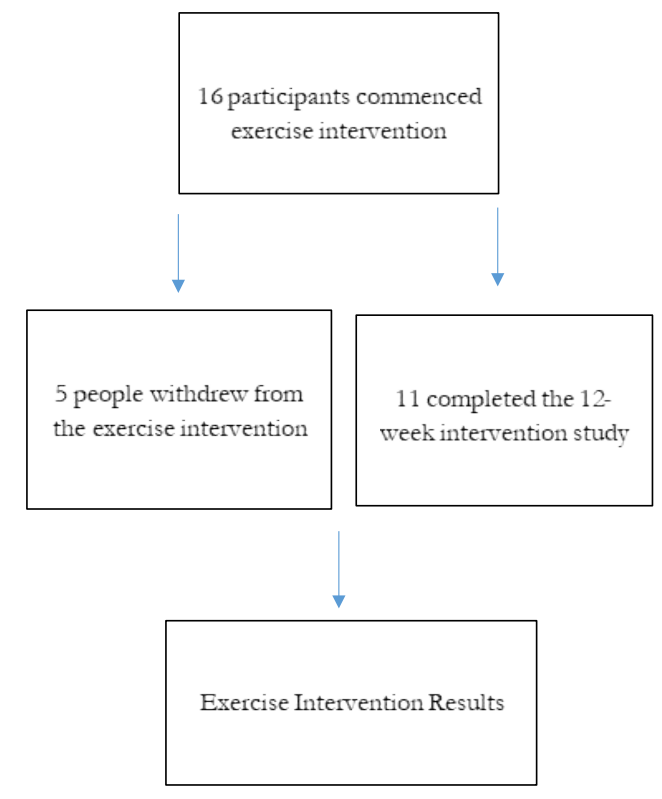

Figure 1. Consort diagram of participants' study inclusion

Table 1. Characteristics of participants at baseline

\begin{tabular}{|c|c|}
\hline Characteristic & $(n=37)$ \\
\hline Age $(y r)$, mean (SD) & $32(7)$ \\
\hline Sex, number male (\%) & $5(45)$ \\
\hline Weight $(\mathrm{kg})$, mean (SD) & $99(20)$ \\
\hline Height $(m)$, mean (SD) & $1.8(5.6)$ \\
\hline BMI $(\mathrm{kg} / \mathrm{m} 2)$, mean $(\mathrm{SD})$ & $32.5(5.7)$ \\
\hline Bench press $1 \mathrm{RM}(\mathrm{kg})$, mean (SD) & $60(13)$ \\
\hline Bench press relative strength (\%), mean (SD) & $59(14)$ \\
\hline Leg press $1 \mathrm{RM}(\mathrm{kg})$, mean $(\mathrm{SD})$ & $189(61)$ \\
\hline Leg press relative strength (\%), mean (SD) & $184(55)$ \\
\hline $\mathrm{SubMaxVO}_{2}(\mathrm{ml} / \mathrm{kg} / \mathrm{min})$, mean (SD) & $23.7(2.3)$ \\
\hline Systolic BP $(m m H g)$, mean (SD) & $134(13)$ \\
\hline Diastolic BP $(m m H g)$, mean (SD) & $76(8)$ \\
\hline Pulse wave velocity $(\mathrm{m} / \mathrm{s})$, mean $(\mathrm{SD})$ & $9.5(2.1)$ \\
\hline DEXA (\% body fat), mean (SD) & $29(8)$ \\
\hline DEXA bone mineral content $(\mathrm{kg})$, mean $(\mathrm{SD})$ & $27.1(0.4)$ \\
\hline
\end{tabular}


Table 2. Mean (SD) for clinical outcomes at each time and difference $(95 \% \mathrm{CI})$ between times $(\mathrm{n}=11)$

\begin{tabular}{|c|c|c|c|}
\hline \multirow[t]{2}{*}{ Clinical outcomes } & \multicolumn{2}{|c|}{ Times } & \multirow{2}{*}{$\begin{array}{c}\begin{array}{c}\text { Difference } \\
\text { between times }\end{array} \\
\begin{array}{c}\text { Week } 12 \text { minus } \\
\text { Week } 0\end{array}\end{array}$} \\
\hline & Week 0 & Week 12 & \\
\hline \multicolumn{4}{|l|}{ Sub-maximal aerobic capacity } \\
\hline $\mathrm{VO}_{2} \operatorname{Maximum}(\mathrm{ml} / \mathrm{kg} / \mathrm{min})$ & $\begin{array}{l}25 \\
(9)\end{array}$ & $\begin{array}{l}30 \\
(9)\end{array}$ & $\begin{array}{c}5 \\
\text { (3 to } 7)\end{array}$ \\
\hline \multicolumn{4}{|l|}{ Strength } \\
\hline 1RM Bench press $(\mathrm{kg})$ & $\begin{array}{l}41 \\
(10)\end{array}$ & $\begin{array}{l}59 \\
(18)\end{array}$ & $\begin{array}{c}17 \\
(10 \text { to } 25)\end{array}$ \\
\hline 1 RM Leg press (kg) & $\begin{array}{l}168 \\
(37)\end{array}$ & $\begin{array}{l}235 \\
(48)\end{array}$ & $\begin{array}{c}67 \\
\text { (36 to } 110)\end{array}$ \\
\hline \multicolumn{4}{|l|}{ Arterial occlusion } \\
\hline Pulse wave velocity $(\mathrm{m} / \mathrm{s})$ & $\begin{array}{l}10.0 \\
(1)\end{array}$ & $\begin{array}{l}8.6 \\
(1)\end{array}$ & $\begin{array}{c}-1.5 \\
-2.3 \text { to }-0.7\end{array}$ \\
\hline \multicolumn{4}{|l|}{ Anthropometry } \\
\hline Waist circumference $(\mathrm{cm})$ & $\begin{array}{c}97 \\
(24)\end{array}$ & $\begin{array}{c}94 \\
(22)\end{array}$ & $\begin{array}{c}-3 \\
(-4 \text { to }-1)\end{array}$ \\
\hline Hip circumference (cm) & $\begin{array}{l}101 \\
(24)\end{array}$ & $\begin{array}{c}98 \\
(23)\end{array}$ & $\begin{array}{c}-2 \\
(-4.4 \text { to }-1.1)\end{array}$ \\
\hline Weight (kg) & $\begin{array}{c}86 \\
(29)\end{array}$ & $\begin{array}{c}94 \\
(28)\end{array}$ & $\begin{array}{c}8 \\
(-20 \text { to } 6)\end{array}$ \\
\hline \multicolumn{4}{|l|}{ Whole body lean mass } \\
\hline Body fat (\%) & $\begin{array}{l}30 \\
(7)\end{array}$ & $\begin{array}{l}28 \\
(7)\end{array}$ & $\begin{array}{c}-2 \\
(-3.4 \text { to }-0.2)\end{array}$ \\
\hline \multicolumn{4}{|l|}{ Brachial blood pressure $(\mathrm{mmHg})$} \\
\hline Systolic & $\begin{array}{l}125 \\
(14)\end{array}$ & $\begin{array}{l}121 \\
(9)\end{array}$ & $\begin{array}{c}-3 \\
\text { (-9 to } 2)\end{array}$ \\
\hline Diastolic & $\begin{array}{l}75 \\
(7)\end{array}$ & $\begin{array}{l}75 \\
(8)\end{array}$ & $\begin{array}{c}-0 \\
(-4 \text { to } 4)\end{array}$ \\
\hline
\end{tabular}

\section{Discussion}

This study found, for the first time, that a supervised exercise intervention can be used with good compliance in community-based Aboriginal adults once they commit to participation and is associated with improvements in aerobic capacity, body fatness and vascular function. These findings suggest that an exercise program based on current ACSM guidelines has the potential to reduce the incidence of non-communicable disease.

The results of the current study supports previous investigations which have suggested that supervised exercise training and/or increased sports participation can improve fitness, waist circumference, and blood pressure [13]. Our results significantly advance these observations by demonstrating that exercise training can benefit other known cardiometabolic risk factors including high body fat and elevated pulse wave velocity. Body fat is considered to be an important modifiable risk factor which is independent of body weight [19], and pulse wave velocity indicates the elasticity, hence the health of an artery [20].

Aerobic capacity is a strong predictor of mortality and is often poor in individuals with chronic disease [21]. Using a combination of supervised aerobic and resistance exercise, our study found a significant mean improvement in aerobic fitness (approximately 19.6\% increase). This compares favorably with previous reports in non-Indigenous people of similar age and baseline fitness, which used aerobic [22] or combined modality exercise interventions [23]. Our observed fitness changes were associated with large improvements in strength on bench press and leg press exercise and a small but statistically insignificant increase (mean $250 \mathrm{~g}$ ) in lean body mass. Numerous studies have shown that an increase in muscle mass is highly protective against the metabolic syndrome risk factors [24] and non-communicable chronic diseases such as diabetes, [25] cancer, [26] heart failure, [27] chronic obstructive pulmonary disease and kidney disease. In the current study, exercise participation also led to significant mean improvement in body fat percentage ( $1.8 \%$ reduction) and waist circumference ( $8.1 \%$ reduction). Together the results demonstrate that increases in cardiovascular fitness, muscular fitness and body composition can be achieved in Aboriginal men and women using current exercise recommendations.

Cardiovascular disease results from several factors which lead to increased systolic blood pressure and pulse pressure because of higher arterial stiffness. Pulse wave velocity (PWV) is considered the gold standard for measuring arterial stiffness [28]. In our study, participants demonstrated elevated PWV, to levels comparable with those seen in older men with mild hypertension [29]. We observed a mean $15 \%$ decrease in PWV following exercise training and a small reduction in diastolic but not systolic blood pressure. This latter outcome is similar to a previous report from exercise-only studies [22], but maybe inferior to blood pressure benefits previously shown in Aboriginal women who undertook a combined exercise and diet intervention.

A limitation of the current study was the relatively poor exercise adoption and subsequent small sample size. Recruitment was constrained by access to fitness centres, which meant that many candidates withdrew interest in participating due to travel requirements for testing and training. While the current study, therefore, provides preliminary evidence concerning the efficacy of exercise for health benefit in Indigenous Australian adults, further studies are required using a larger cohort (partly via improving access to facilities) and acquiring a full suite of cardio-metabolic outcome measures e.g. lipids and lipoproteins. In addition to the observed physiological benefits, our study also provides useful first information concerning the implementation of culturally competent exercise programs in a metropolitan setting. We observed high compliance (over 90\%) with the supervised exercise training, in individuals who arguably wouldn't obtain a fitness centre membership due to either the financial burden or the perception of the fitness centre environment as a "culturally unsafe" place. The improved understanding of cultural safety may have been achieved by the promotion of communication between the Indigenous researcher, the participants and non-Indigenous users of the fitness centre, and the use of the group training approach in a traditionally non-Indigenous environment. All participants who completed the trial reported better exercise knowledge and wanted to continue the program after study completion.

Previous studies in Indigenous Australian communities have suggested positive benefits from exercise interventions including improved body weight, BMI and blood pressure, albeit with generally poor compliance to exercise classes [13]. In the current study, we assessed the effect of regular supervised exercise on cardiovascular fitness, body composition and arterial stiffness.

\section{Conclusion}

This is the first study to recruit Indigenous male and female participants for an exercise intervention based on current guidelines for physical activity and involving close supervision in a metropolitan fitness centre setting. Numerous measures were used that have not been previously examined in the Indigenous community including DEXA and Pulse Wave Velocity for the in-depth evaluation of body composition and central cardiovascular function. We demonstrated that combined aerobic and resistance training led to significant improvements in aerobic fitness, body composition and pulse wave 
velocity. The outcome is that supervised exercise can be implemented with high compliance, in a culturally appropriate way in a fitness centre setting and may be used to reduce the risk of non-communicable disease in Indigenous Australian adults. Future research should aim to examine the effect of exercise on other known cardiovascular risk factors (including lipids and lipoproteins), and on strategies to promote increased adoption of exercise in Indigenous Australians.

\section{Practical implications}

- This study demonstrates that a gym can be a culturally appropriate place for Indigenous Australians.

- The gym is an effective and readily available therapy for Indigenous health improvement

- The study reports for the first time in the Australian Indigenous community gold standard physiological measures which may be used to compare with non-Indigenous and other International Indigenous communities.

\section{Ethical considerations}

The protocol was fully explained to each subject and written informed consent was obtained from all individuals prior to their participation in the study. This study was approved by the Edith Cowan University Human Ethics Committee and Western Australian Aboriginal Health Information and Ethics Committee (WAAHIEC). The funding bodies had no role in the collection, analysis, interpretation and did not have the right to apply or disapprove publication of this finished manuscript.

\section{References}

1. Australian Institute of Health and Welfare (2008) Health-car expenditure on cardiovascular diseases 2008-2009.

2. Anderson I, Crengle S, Kamaka ML, Chen TH, Palafox N, et al. (2006) Indigenous health in Australia, New Zealand, and the Pacific. The Lancet 367: 1775-1785.

3. Mendis S, Puska P, Norrving B (2011) Global atlas on cardiovascular disease prevention and control, World Health Organization.

4. Zhao Y, Condon J, Lawton P, He V, Cadilhac DA, et al. (2016) Lifetime direct costs of stroke for indigenous patients adjusted for comorbidities. Neurology 87: 458-465.

5. Dwyer J, Kelly J, Willis E, Mackean T, Battersby M, et al. (2011) Managing Two Worlds Together: Study 2 -Staff Perspectives on Care for Country Aboriginal Patients.

6. Thompson PD, Buchner D, Pina IL, Balady GJ, Williams MA, et al. (2003) Exercise and physical activity in the prevention and treatment of atherosclerotic cardiovascular disease: a statement from the Council on Clinical Cardiology (Subcommittee on Exercise, Rehabilitation, and Prevention) and the Council on Nutrition, Physical Activity, and Metabolism (Subcommittee on Physical Activity). Circulation 107: 3109-3116. [Crossref]

7. Australian Institute of Health and Welfare (2016) Australian Burden of Disease Study: Impact and causes of illness and death in Australia 2011. In: Welfare. AIoHa, (Edn). Canberra: AHIW

8. Reeves MM, Terranova CO, Eakin EG, Demark-Wahnefried W (2014) Weight loss intervention trials in women with breast cancer: a systematic review. Obes Rev 15: 749-768. [Crossref]

9. Ezzati M, Riboli E (2013) Behavioral and dietary risk factors for noncommunicable diseases. NEW ENGL J MED 369: 954-964.
10. Taylor G, McNeill A, Girling A, Farley A, Lindson-Hawley N, et al. (2014) Change in mental health after smoking cessation: systematic review and meta-analysis. $B M J$ 348: g1151.

11. Hadi HA, Carr CS, Suwaidi JA (2005) Endothelial dysfunction: cardiovascular risk factors, therapy, and outcome. Vasc Health Risk Manag 1: 183. [Crossref]

12. Rowley KG, Daniel M, Skinner K, Skinner M, White GA, et al. (2000) Effectiveness of a community-directed 'healthy lifestyle' program in a remote Australian aboriginal community. AUST NZ J PUBL HEAL 24: 136-144.

13. Canuto K, Cargo M, Li M, D’Onise K, Esterman A, et al. (2012) Pragmatic randomised trial of a 12-week exercise and nutrition program for Aboriginal and Torres Strait Islander women: clinical results immediate post and 3 months follow-up. BMC Public Health 12: 933. [Crossref]

14. Mendham A, Duffield R, Marino F, Coutts A (2014) A 12 week sports-based exercise programme for inactive Indigenous Australian men improved clinicl risk factors associated with type 2 diabetes mellitus. J SCI MED SPORT.

15. Thomson N, Kirov E (2006) Summary of Indigenous Health: Preliminary Analysis of Physical Activity. Aboriginal and Islander Health Worker Journal 30: 14 -16.

16. Zhang L, Shi L, Morrison JA (2016) Healthy Lifestyle Factors and Change in Adults' Cardiometabolic Health. Health Behavior and Policy Review 3: 488-498.

17. American College of Sports Medicine. Appropriate physical activity intervention strategies for weight loss and prevention of weight regain for adults.

18. Suleman R, Padwal R, Hamilton P, Senthilselvan A, Alagiakrishnan K (2017) Association between central blood pressure, arterial stiffness, and mild cognitive impairment. Clinical Hypertension 23: 2.

19. Romero-Corral A, Montori VM, Somers VK, Korinek J, Thomas RJ, et al. (2006) Association of bodyweight with total mortality and with cardiovascular events in coronary artery disease: a systematic review of cohort studies. The Lancet 368: 666678

20. AlGhatrif M, Wang M, Fedorova OV (2017) The Pressure of Aging. Medical Clinics of North America. 101: 81-101.

21. Greely SJ, Martinez N, Campbell BI (2013) The high impact of high-intensity interval training on metabolic syndrome. STRENGTH COND J 35: 63-65.

22. Fagard RH (2006) Exercise is good for your blood pressure: effects of endurance training and resistance training. CLIN EXP PHARMACOL P 33: 853-856.

23. Davidson L, Hudson R, Kilpatrick K, Kuk JL, McMillan K, et al. (2009) Effects of exercise modality on insulin resistance and functional limitations in older adults. Archive of Internal Medicine 169: 122-131.

24. Leenders M, Verdijk LB, van der Hoeven L, Adam J, van Kranenburg J, et al. (2013) Patients With Type 2 Diabetes Show a Greater Decline in Muscle Mass, Muscle Strength, and Functional Capacity With Aging. J AM MED DIR ASSOC 14: 585-592.

25. Kalyani RR, Corriere M, Ferrucci L (2014) Age-related and disease-related muscle loss: the effect of diabetes, obesity, and other diseases. Lancet Diabetes Endocrinol 2: 819-829. [Crossref]

26. Pedersen BK, Febbraio MA (2012) Muscles, exercise and obesity: skeletal muscle as a secretory organ. Nat Rev Endocrinol 8: 457-465. [Crossref]

27. Fulster S, Tacke M, Sandek A, Ebner N, Tschöpe C, et al. (2013) Muscle wasting in patients with chronic heart failure: results from the studies investigating co-morbidities aggravating heart failure (SICA-HF). EUR HEART J 34: 512-519.

28. Janner JH, Godtfredsen NS, Ladelund S, Vestbo J, Prescott E (2012) The association between aortic augmentation index and cardiovascular risk factors in a large unselected population. J HUM HYPERTENS 26: 476-484.

29. Collier SR, Kanaley JA, Carhart R, Jr., Frechette V, Tobin MM, et al. (2008) Effect of 4 weeks of aerobic or resistance exercise training on arterial stiffness, blood flow and blood pressure in pre- and stage-1 hypertensives. J Hum Hypertens 22: 678-686. [Crossref]

Copyright: (C2019 Esgin T. This is an open-access article distributed under the terms of the Creative Commons Attribution License, which permits unrestricted use, distribution, and reproduction in any medium, provided the original author and source are credited. 